\title{
Topological Charge of (Lattice) Gauge Fields
}

\author{
M. Göckeler ${ }^{1}$, M. L. Laursen ${ }^{2}$, G. Schierholz ${ }^{3,4}$, and U.-J. Wiese ${ }^{5}$ \\ ${ }^{1}$ Institut für Theoretische Physik der Universität, D-6900 Heidelberg, Federal Republic of \\ Germany \\ ${ }^{2}$ NORDITA, Copenhagen, Denmark \\ ${ }^{3}$ Institut für Theoretische Physik der Universität, D-2300 Kiel, Federal Republic of Germany \\ ${ }^{4}$ Deutsches Elektronen-Synchrotron DESY, D-2000 Hamburg, Federal Republic of Germany \\ ${ }^{5}$ Institut für Theoretische Physik der Universität, D-3000 Hannover, Federal Republic of \\ Germany
}

\begin{abstract}
Using recently derived explicit formulae for the 2- and 3-cochains in SU(2) gauge theory, we are able to integrate the Chern-Simons density analytically. We arrive - in SU(2) - at a local algebraic expression for the topological charge, which is the sum of local winding numbers associated with the corners (lattice points) of the cells covering the manifold plus contributions from possible isolated gauge singularities which manifest themselves as "vortices" in the 1-, 2- or 3-cochains. Among others we consider hypercubic geometry - i.e. covering the manifold by hypercubes - which is of particular interest to lattice Monte Carlo applications. Finally, we extend our results to SU(3) gauge theory.
\end{abstract}

\section{Introduction}

Differentiable $\mathrm{SU}(N)$ gauge fields on a compact 4-manifold $\mathbb{M}$ carry a topological charge [1]

$$
Q=-\frac{1}{32 \pi^{2}} \int_{\mathbb{M}} d^{4} x \varepsilon_{\mu v \varrho \sigma} \operatorname{Tr}\left[F_{\mu v} F_{\varrho \sigma}\right],
$$

where

$$
F_{\mu v}=\partial_{\mu} A_{v}-\partial_{v} A_{\mu}+\left[A_{\mu}, A_{v}\right] .
$$

The charge $Q$ is a measure for topologically non-trivial properties of the gauge fields, which have been argued to play an important role in the physics of the vacuum of $\mathrm{QCD}$ and $\mathrm{SU}(N)$ gauge theories.

Preliminary results of calculations of $Q$ in SU(2) gauge theory on the lattice $[2,3]$ hold out hope for a quantitative resolution of the U(1) problem [4]. The recent finding [5] that the vacuum of the quantized (pure) SU(2) gauge theory possesses an underlying instanton structure brings us furthermore in touch with semi-classical ideas of the QCD vacuum [6] and a possible mechanism for chiral symmetry breaking [7], which could be the beginning of a better understanding of the non-perturbative phenomena of these theories. 
So far these investigations have been limited to smaller lattices and hence to smaller values of $\beta$. The difficulty involved is that a meaningful transcription of the topological charge (1), as it stands, onto the (periodic) lattice [8] is mathematically voluminous and very time consuming to compute, since the transition functions and their derivatives must be constructed everywhere on the boundary of each hypercube.

We realize, that the 4-dimensional integral in Eq. (1) can be performed analytically with the help of the cochain reduction given in [9]. The outcome is a local algebraic expression for the topological charge (1) in the continuum, which is relatively easy to implement on the lattice and fast to compute. The derivation of this result, which we believe is also of interest beyond the scope of lattice gauge theory applications as it allows a simple geometrical interpretation of topology, will be given in the present paper.

The paper is organized as follows. Sections II and III deal with the continuum: in Sect. II we do the integral for SU(2) gauge fields covering the 4-manifold $\mathbb{M}$ by a "generic" set of cells, while in Sect. III we take $\mathbb{M}$ to be the 4-torus and cover it by hypercubes. In Sect. IV we briefly outline what it involves to evaluate the new expression for $Q$ on the lattice. In Sect. $\mathrm{V}$ we extend our results to gauge group SU(3). We finish with some concluding remarks in Sect. VI.

\section{Continuum SU(2) Gauge Fields and Generic Geometry}

The derivation in this section will follow the cochain reduction of the ChernSimons density given in [9].

We cover the compact manifold $\mathbb{M}$ by a set of cells $c_{i}$, i.e.

$$
\mathbb{M}=\bigcup_{i} c_{i}
$$

with

$$
c_{i} \cap c_{j}=\partial c_{i} \cap \partial c_{j}
$$

The cells are chosen such that $5-n$ cells overlap in a (possibly empty) $n$-dimensional intersection. This we refer to as generic geometry. The topological charge (1) can then be written

$$
Q=-\frac{1}{32 \pi^{2}} \sum_{i} \int_{c_{\imath}} d^{4} x \varepsilon_{\mu \nu \varrho \sigma} \operatorname{Tr}\left[F_{\mu \nu}^{i} F_{\varrho \sigma}^{i}\right],
$$

where the index $i$ on the fields refers to the gauge in the cell $c_{i}$. In each cell we may gauge transform the gauge fields into a complete axial gauge by

$$
\tilde{A}_{\mu}^{i}=g_{i}^{-1}\left(A_{\mu}^{i}+\partial_{\mu}\right) g_{i} .
$$

Making use of the fact that the Chern-Pontryagin density is a total divergence, i.e.

$$
P=-\frac{1}{32 \pi^{2}} \varepsilon_{\mu \nu \rho \sigma} \operatorname{Tr}\left[F_{\mu \nu}^{i} F_{\varrho \sigma}^{i}\right]=\partial_{\mu} \Omega_{\mu}^{(0)}(i),
$$

with

$$
\Omega_{\mu}^{(0)}(i)=-\frac{1}{8 \pi^{2}} \varepsilon_{\mu \nu \varrho \sigma} \operatorname{Tr}\left[\tilde{A}_{v}^{i}\left(\partial_{\varrho} \tilde{A}_{\sigma}^{i}+\frac{2}{3} \tilde{A}_{\varrho}^{i} \tilde{A}_{\sigma}^{i}\right)\right]
$$


we obtain

$$
Q=\sum_{i} \int_{\partial c_{\imath}} d^{3} \sigma_{\mu} \Omega_{\mu}^{(0)}(i)=\frac{1}{2 !} \sum_{i, j} \int_{c_{\imath} \cap c_{j}} d^{3} \sigma_{\mu}\left(\Omega_{\mu}^{(0)}(i)-\Omega_{\mu}^{(0)}(j)\right),
$$

where $c_{i} \cap c_{j}$ has the orientation of $\partial c_{i}$.

We introduce transition functions relating the gauges $i, j$ in the cells $c_{i}, c_{j}$ on $c_{i} \cap c_{j}$ by

$$
\tilde{A}_{\mu}^{j}=v_{i j}^{-1}\left(\tilde{A}_{\mu}^{i}+\partial_{\mu}\right) v_{i j} .
$$

Comparing this with Eq. (6) one finds (on $c_{i} \cap c_{j}$ )

$$
v_{i j}=g_{i}^{-1} g_{j} \text {. }
$$

We recognize now that the integrand on the right-hand side of Eq. (9) is the coboundary operation

$$
\Omega_{\mu}^{(0)}(i)-\Omega_{\mu}^{(0)}(j)=\Delta \Omega_{\mu}^{(0)}(i, j)=\partial_{\nu} \Omega_{\mu \nu}^{(1)}(i, j),
$$

which is again a total divergence. This excludes singular points in the interior of $c_{i} \cap c_{j}$, i.e.

$$
x \in\left(c_{i} \cap c_{j}\right) \backslash \partial\left(c_{i} \cap c_{j}\right),
$$

at which

$$
v_{i j}=\exp i \alpha \tau=\cos \alpha+i \sin \alpha \mathbf{e}_{\alpha} \tau, \quad \alpha \in[0, \pi]
$$

becomes -1 , i.e. $\alpha=\pi$, where [9]

$$
\begin{aligned}
\Omega_{\mu \nu}^{(1)}(i, j)= & -\frac{1}{8 \pi^{2}}(\alpha-\sin \alpha \cos \alpha) \varepsilon_{\mu \nu \varrho \sigma} \mathbf{e}_{\alpha} \cdot\left(\partial_{\varrho} \mathbf{e}_{\alpha} \times \partial_{\sigma} \mathbf{e}_{\alpha}\right) \\
& -\frac{1}{8 \pi^{2}} \varepsilon_{\mu \nu \varrho \sigma} \operatorname{Tr}\left[\partial_{\varrho} v_{i j} v_{i j}^{-1} \tilde{A}_{\sigma}^{i}\right]
\end{aligned}
$$

has a "vortex"

$$
-\frac{1}{8 \pi} \varepsilon_{\mu \nu \varrho \sigma} \mathbf{e}_{\alpha} \cdot\left(\partial_{\varrho} \mathbf{e}_{\alpha} \times \partial_{\sigma} \mathbf{e}_{\alpha}\right)
$$

Applying Gauss' theorem, Eq. (9) then reduces to

$$
Q=Q^{(1)}+Q_{\Sigma}^{(1)}
$$

with

$$
\begin{aligned}
Q_{\Sigma}^{(1)} & =\frac{1}{2 !} \sum_{i, j} \int_{\partial\left(c_{i} \cap c_{j}\right)} d^{2} \sigma_{\mu \nu} \Omega_{\mu \nu}^{(1)}(i, j) \\
& =\frac{1}{3 !} \sum_{i, j, k} \int_{c_{i} \cap c_{j} \cap c_{k}} d^{2} \sigma_{\mu \nu}\left[\Omega_{\mu \nu}^{(1)}(i, j)-\Omega_{\mu \nu}^{(1)}(i, k)+\Omega_{\mu \nu}^{(1)}(j, k)\right]
\end{aligned}
$$

and

$$
Q^{(1)}=\frac{1}{2 !} \sum_{i, j} \sum_{x \in\left(c_{i} \cap c_{j}\right) \backslash \partial\left(c_{i} \cap c_{j}\right)} \int_{S_{\varepsilon}^{2}(x)} d^{2} \sigma_{\mu \nu} \frac{1}{8 \pi} \varepsilon_{\mu \nu \varrho \sigma} \mathbf{e}_{\alpha} \cdot\left(\partial_{\varrho} \mathbf{e}_{\alpha} \times \partial_{\sigma} \mathbf{e}_{\alpha}\right)
$$


where $S_{\varepsilon}^{2}(x)$ is a sphere of radius $\varepsilon$ around $x$ and where $c_{i} \cap c_{j} \cap c_{k}$ and $S_{\varepsilon}^{2}(x)$ have the orientation of $\partial\left(c_{i} \cap c_{j}\right)$.

The integral in Eq. (19) gives

$$
\int_{S_{\varepsilon}^{2}(x)} d^{2} \sigma_{\mu \nu} \frac{1}{8 \pi} \varepsilon_{\mu v \varrho \sigma} \mathbf{e}_{\alpha} \cdot\left(\partial_{\varrho} \mathbf{e}_{\alpha} \times \partial_{\sigma} \mathbf{e}_{\alpha}\right)=n^{(1)}(x ; i, j)
$$

where $n^{(1)}$ is the $S^{2}$ winding number associated with the singularity at $x$. In the vicinity of $x$ we can write

$$
v_{i j}=-1+i M_{\alpha \beta} d x_{\alpha} \tau_{\beta}+\ldots,
$$

which for $\operatorname{det} M \neq 0$ results in

$$
n^{(1)}(x ; i, j)=\operatorname{sign}(\operatorname{det} M)= \pm 1
$$

In total we then obtain

$$
Q^{(1)}=\sum_{\Lambda^{(1)}} n^{(1)}(x ; i, j)
$$

where the sum is over all singular points $x$, i.e.

$$
\Lambda^{(1)}=\bigcup_{i, j}\left\{x \in\left(c_{i} \cap c_{j}\right) \backslash \partial\left(c_{i} \cap c_{j}\right)\right\} .
$$

From Eq. (11) we derive the cocycle condition

which, writing

$$
v_{i j} v_{j k}=v_{i k}
$$

$$
v_{i j}=\exp (i \alpha \tau), \quad v_{j k}=\exp (i \beta \tau), \quad v_{i k}=\exp (i \gamma \tau),
$$

defines a spherical triangle as has been shown in [9]. The integrand on the righthand side of Eq. (18) is the coboundary operation

$$
\Omega_{\mu \nu}^{(1)}(i, j)-\Omega_{\mu \nu}^{(1)}(i, k)+\Omega_{\mu \nu}^{(1)}(j, k)=\Delta \Omega_{\mu \nu}^{(1)}(i, j, k)=\partial_{\varrho} \Omega_{\mu \nu \varrho}^{(2)}(i, j, k),
$$

which again is a total divergence. This excludes singular points

at which

$$
x \in\left(c_{i} \cap c_{j} \cap c_{k}\right) \backslash \partial\left(c_{i} \cap c_{j} \cap c_{k}\right),
$$

$$
(\boldsymbol{\alpha}+\boldsymbol{\beta}-\boldsymbol{\gamma}) \mathbf{e}_{\alpha}=(\boldsymbol{\alpha}+\boldsymbol{\beta}-\boldsymbol{\gamma}) \mathbf{e}_{\beta}=-(\boldsymbol{\alpha}+\boldsymbol{\beta}-\boldsymbol{\gamma}) \mathbf{e}_{\gamma}=-2 \pi,
$$

i.e. where the spherical triangle defined by the transition functions (26) degenerates to a circle, where [9]

$$
\begin{aligned}
\Omega_{\mu \nu \varrho}^{(2)}(i, j, k)=- & \frac{1}{8 \pi^{2}} \varepsilon_{\mu \nu \varrho \sigma}\left(1+2 \cos \alpha \cos \beta \cos \gamma-\cos ^{2} \alpha-\cos ^{2} \beta-\cos ^{2} \gamma\right)^{-1} \\
& \cdot\left\{( \boldsymbol { \alpha } + \boldsymbol { \beta } - \gamma ) \cdot ( \operatorname { s i n } \alpha \mathbf { e } _ { \alpha } ) \left[\partial_{\sigma}\left(\sin \beta \mathbf{e}_{\beta}\right)\right.\right. \\
& \left.\cdot\left(\sin \gamma \mathbf{e}_{\gamma}\right)-\left(\sin \beta \mathbf{e}_{\beta}\right) \cdot \partial_{\sigma}\left(\sin \gamma \mathbf{e}_{\gamma}\right)\right] \\
+ & (\boldsymbol{\alpha}+\boldsymbol{\beta}-\gamma) \cdot\left(\sin \beta \mathbf{e}_{\beta}\right)\left[\partial_{\sigma}\left(\sin \gamma \mathbf{e}_{\gamma}\right)\right. \\
& \left.\cdot\left(\sin \alpha \mathbf{e}_{\alpha}\right)-\left(\sin \gamma \mathbf{e}_{\gamma}\right) \cdot \partial_{\sigma}\left(\sin \alpha \mathbf{e}_{\alpha}\right)\right] \\
+ & (\alpha+\boldsymbol{\beta}-\gamma) \cdot\left(\sin \gamma \mathbf{e}_{\gamma}\right)\left[\partial_{\sigma}\left(\sin \alpha \mathbf{e}_{\alpha}\right)\right. \\
& \left.\left.\cdot\left(\sin \beta \mathbf{e}_{\beta}\right)-\left(\sin \alpha \mathbf{e}_{\alpha}\right) \cdot \partial_{\sigma}\left(\sin \beta \mathbf{e}_{\beta}\right)\right]\right\}
\end{aligned}
$$


has a "vortex"

$$
\begin{gathered}
-\frac{1}{4 \pi^{2}} \varepsilon_{\mu \nu \varrho \sigma} \frac{(\boldsymbol{\alpha}+\boldsymbol{\beta}-\boldsymbol{\gamma}) \cdot\left(\partial_{\sigma} \mathbf{S} \times \mathbf{S}\right)}{S^{2}}=-\frac{1}{2 \pi} \varepsilon_{\mu \nu \varrho \sigma} \frac{\mathbf{e}_{\alpha} \cdot\left(\partial_{\sigma} \boldsymbol{\delta} \times \boldsymbol{\delta}\right)}{\delta^{2}}, \\
\mathbf{S}=\cos \alpha \sin \beta \sin \gamma \mathbf{e}_{\beta} \times \mathbf{e}_{\gamma}+\cos \beta \sin \gamma \sin \alpha \mathbf{e}_{\gamma} \times \mathbf{e}_{\alpha} \\
+\cos \gamma \sin \alpha \sin \beta \mathbf{e}_{\alpha} \times \mathbf{e}_{\beta}, \quad \boldsymbol{\delta}=\mathbf{e}_{\alpha}-\mathbf{e}_{\beta} .
\end{gathered}
$$

The vector $\mathbf{S}$ defines the orientation of the great sphere (in group space) spanned by the transition functions (26) and winds around by $2 \pi$ as one runs round the singularity.

Applying Gauss' theorem, Eq. (18) then reduces to

with

$$
Q_{\Sigma}^{(1)}=Q^{(2)}+Q_{\Sigma}^{(2)}
$$

$$
\begin{aligned}
Q_{\Sigma}^{(2)}= & \frac{1}{3 !} \sum_{i, j, k} \int_{\partial\left(c_{i} \cap c_{j} \cap c_{k}\right)} d \sigma_{\mu \nu \varrho} \Omega_{\mu \nu \varrho}^{(2)}(i, j, k) \\
= & \frac{1}{4 !} \sum_{i, j, k} \int_{c_{2} \cap c_{j} \cap c_{k} \cap c_{l}} d \sigma_{\mu \nu \varrho}\left[\Omega_{\mu \nu \varrho}^{(2)}(i, j, k)-\Omega_{\mu \nu \varrho}^{(2)}(i, j, l)\right. \\
& \left.+\Omega_{\mu \nu \varrho}^{(2)}(i, k, l)-\Omega_{\mu \nu \varrho}^{(2)}(j, k, l)\right]
\end{aligned}
$$

and

$$
Q^{(2)}=\frac{1}{3 !} \sum_{i, j, k} \sum_{x \in\left(c_{\imath} \cap c_{j} \cap c_{k}\right) \backslash \partial\left(c_{\imath} \cap c_{j} \cap c_{k}\right)} \int_{S_{\varepsilon}^{1}(x)} d \sigma_{\mu \nu \varrho} \frac{1}{2 \pi} \varepsilon_{\mu \nu \varrho \sigma} \frac{\mathbf{e}_{\alpha} \cdot\left(\partial_{\sigma} \boldsymbol{\delta} \times \boldsymbol{\delta}\right)}{\delta^{2}},
$$

where $S_{\varepsilon}^{1}(x)$ is a circle of radius $\varepsilon$ around $x$, and where $c_{i} \cap c_{j} \cap c_{k} \cap c_{l}$ and $S_{\varepsilon}^{1}(x)$ have the orientation of $\partial\left(c_{i} \cap c_{j} \cap c_{k}\right)$.

One realizes that the integral in Eq. (34) gives

$$
\int_{S_{\varepsilon}^{1}(x)} d \sigma_{\mu v \varrho} \frac{1}{2 \pi} \varepsilon_{\mu \nu \varrho \sigma} \frac{\mathbf{e}_{\alpha} \cdot\left(\partial_{\sigma} \boldsymbol{\delta} \times \boldsymbol{\delta}\right)}{\delta^{2}}=n^{(2)}(x ; i, j, k),
$$

where $n^{(2)}$ is the $S^{1}$ winding number associated with the singularity $x$ which assumes the values

$$
n^{(2)}(x ; i, j, k)= \pm 1
$$

depending on whether $\mathbf{S}, \boldsymbol{\delta}$ wind around in the positive or negative sense. We then obtain

with

$$
Q^{(2)}=\sum_{\Lambda^{(2)}} n^{(2)}(x ; i, j, k)
$$

$$
\Lambda^{(2)}=\bigcup_{i, j, k}\left\{x \in\left(c_{i} \cap c_{j} \cap c_{k}\right) \backslash \partial\left(c_{i} \cap c_{j} \cap c_{k}\right)\right\}
$$

Expression (33) brings together 4 spherical triangles, which build a spherical tetrahedron as illustrated in [9].

The descent continues:

$$
\begin{aligned}
& \Omega_{\mu v \varrho}^{(2)}(i, j, k)-\Omega_{\mu v \varrho}^{(2)}(i, j, l)+\Omega_{\mu v \varrho}^{(2)}(i, j, l)-\Omega_{\mu \nu \varrho}^{(2)}(j, k, l) \\
& \quad=\Delta \Omega_{\mu \nu \varrho}^{(2)}(i, j, k, l)=\partial_{\sigma} \Omega_{\mu \nu \varrho \sigma}^{(3)}(i, j, k, l) .
\end{aligned}
$$


In [9] we have shown that

$$
\Omega_{\mu \nu \varrho \sigma}^{(3)}(i, j, k, l)=\frac{1}{2 \pi^{2}} \varepsilon_{\mu \nu \varrho \sigma} V(i, j, k, l),
$$

where $V(i, j, k, l)$ is the volume of the spherical tetrahedron defined by the transition functions relating the gauges $i, j, k, l$. We realize that $V(i, j, k, l)$ has a discontinuity at points

$$
x \in\left(c_{i} \cap c_{j} \cap c_{k} \cap c_{l}\right) \backslash \partial\left(c_{i} \cap c_{j} \cap c_{k} \cap c_{l}\right),
$$

where the spherical tetrahedron degenerates to a sphere. Applying Gauss' theorem we then obtain two contributions to Eq. (33):

$$
Q_{\Sigma}^{(2)}=Q^{(3)}+Q_{\Sigma}^{(3)}
$$

The first contribution gives

$$
Q^{(3)}=\sum_{\Lambda^{(3)}} n^{(3)}(x ; i, j, k, l)
$$

with

$$
\Lambda^{(3)}=\bigcup_{i, j, k, l}\left\{x \in\left(c_{i} \cap c_{j} \cap c_{k} \cap c_{l}\right) \backslash \partial\left(c_{i} \cap c_{j} \cap c_{k} \cap c_{l}\right)\right\},
$$

where $n^{(3)}$ accounts for the change of the orientation of the spherical tetrahedron at the point $x$, i.e.

$$
n^{(3)}(x ; i, j, k, l)=\left.\frac{1}{2 \pi^{2}} \varepsilon_{\mu v \varrho \sigma} \Delta V(i, j, k, l)\right|_{x}= \pm 1 .
$$

For the second contribution we obtain

$$
\begin{aligned}
Q_{\Sigma}^{(3)}= & \frac{1}{4 !} \sum_{\partial\left(c_{i} \cap c_{j} \cap c_{k} \cap c_{l}\right)} \sigma \frac{1}{2 \pi^{2}} V(i, j, k, l) \\
= & \frac{1}{5 !} \sum_{c_{i} \cap c_{j} \cap c_{k} \cap c_{l} \cap c_{m}} \sigma \frac{1}{2 \pi^{2}}[V(i, j, k, l)-V(i, j, k, m) \\
& +V(i, j, l, m)-V(i, k, l, m)+V(j, k, l, m)],
\end{aligned}
$$

where $\sigma= \pm 1$ is a sign factor, which denotes the orientation of $\partial\left(c_{i} \cap c_{j} \cap c_{k} \cap c_{l}\right)$.

The intersection of 54-dimensional cells defines a point which naturally leads to the notation of the "lattice":

$$
\Lambda=\bigcup_{i, j, k, l, m} c_{i} \cap c_{j} \cap c_{k} \cap c_{l} \cap c_{m} .
$$

Thus we can rewrite Eq. (46) in the form

with

$$
Q_{\Sigma}^{(3)}=\sum_{\Lambda} n(i, j, k, l, m)
$$

$$
\begin{aligned}
n(i, j, k, l, m)= & \frac{\sigma}{2 \pi^{2}}[V(i, j, k, l)-V(i, j, k, m)+V(i, j, l, m) \\
& -V(i, k, l, m)+V(j, k, l, m)]
\end{aligned}
$$



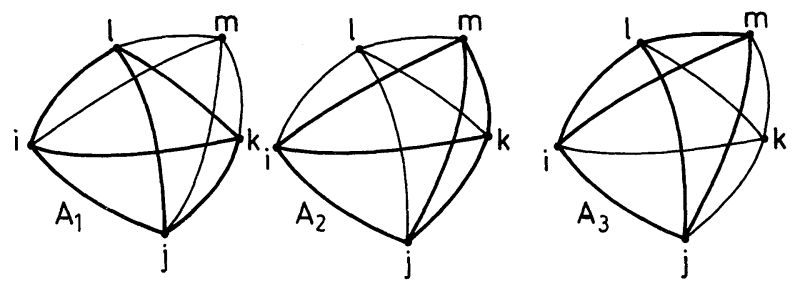

(a)

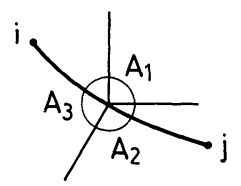

(b)

Fig. 1. The 5 spherical tetrahedra covering $S^{3} . A_{1}$ is the angle between the spherical triangles $(i, j, k)$ and $(i, j, l), A_{2}$ is the angle between the spherical triangles $(i, j, k)$ and $(i, j, m)$ and $A_{3}$ is the angle between the spherical triangles $(i, j, m)$ and $(i, j, l)$

Note that the 1/5! factor in Eq. (46) has disappeared because of the implicit summation over all permutations of cells. As we have discussed in [9], $n$ combines 5 spherical tetrahedra, which wind around $S^{3}$, the group space of SU(2). Since the volume of $S^{3}$ is $2 \pi^{2}$ and the 5 spherical tetrahedra together are compact and so cover $S^{3}$ (but at most once), we find

$$
n=0, \pm 1 \text {. }
$$

The calculation of $n(i, j, k, l, m)$ proceeds as follows. We take the transition functions $v_{i j}, v_{j k}, \ldots$ and determine the angles between adjacent spherical triangles intersecting along a "hinge" of the spherical tetrahedra (cf. [9]). In Fig. 1a we have illustrated this for a particular "hinge" with which are associated 3 angles, $A_{1}, A_{2}$, and $A_{3}$, belonging to 3 different spherical tetrahedra. For geometrical reasons

$$
\frac{\left(A_{1}+A_{2}+A_{3}\right)}{2 \pi} \equiv p=0, \pm 1,
$$

a special example of which is given in Fig. $1 \mathrm{~b}$. If one of the $10 p$ 's is zero, then evidently $n=0$. If all $p$ 's are +1 , then $n=+1$, and if all $p$ 's are -1 , we will have $n=-1$.

Collecting our results now, we obtain for the topological charge

$$
\begin{aligned}
Q= & \sum_{\Lambda} n(i, j, k, l, m)+\sum_{\Lambda^{(1)}} n^{(1)}(x ; i, j) \\
& +\sum_{\Lambda^{(2)}} n^{(2)}(x ; i, j, k)+\sum_{\Lambda^{(3)}} n^{(3)}(x ; i, j, k, l),
\end{aligned}
$$

which is the sum of local winding numbers and, consequently, assumes integer values. Expression (52) is gauge invariant by construction. It furthermore has a simple, geometrical interpretation. 
In the continuum we can arrange that neither $v_{i, j}=-1$ in $\left(c_{i} \cap c_{j}\right) \backslash \partial\left(c_{i} \cap c_{j}\right)$, $(\boldsymbol{\alpha}+\boldsymbol{\beta}-\gamma) \mathbf{e}_{\alpha}=2 \pi$ in $\left(c_{i} \cap c_{j} \cap c_{k}\right) \backslash \partial\left(c_{i} \cap c_{j} \cap c_{k}\right)$ nor $\left.\Delta V(i, j, k, l)\right|_{x}= \pm 2 \pi^{2}$ at $x \in\left(c_{i} \cap c_{j} \cap c_{k} \cap c_{l}\right) \backslash \partial\left(c_{i} \cap c_{j} \cap c_{k} \cap c_{l}\right)$, in which case the topological charge takes the simple form

$$
Q=\sum_{\Lambda} n(i, j, k, l, m)
$$

For a further discussion see Sect. IV.

\section{Hypercubic Geometry}

So far we have considered a generic geometry of cells covering $\mathbb{M}$. Now we come to the case of a hypercubic geometry, which is of special interest to lattice gauge theory calculations, and where $\mathbb{M}$ is the 4-torus $T^{4}$.

The "lattice" is now defined by

$$
\Lambda=\left\{s \in \mathbb{M} \mid s_{\mu} \in \mathbb{Z}, \mu=0,1,2,3\right\} .
$$

The cells covering $\mathbb{M}$ are the hypercubes (of length 1),

$$
\mathbb{M}=\bigcup_{s \in \Lambda} c(s)
$$

with

$$
c(s)=\left\{y \in \mathbb{M} \mid s_{\mu} \leqq y_{\mu} \leqq s_{\mu}+1\right\} .
$$

The topological charge (1) can then be written

$$
Q=\sum_{s \in \Lambda} \int_{c(s)} d^{4} x P
$$

where $P$ is the Chern-Pontryagin density given in Eq. (7). Using

$$
P=\partial_{\mu} \Omega_{\mu}^{(0)}(s),
$$

we can perform one integration as before and obtain

$$
Q=\sum_{s \in A} \sum_{\mu} \int_{f(s, \mu)} d^{3} \sigma_{\mu}\left[\Omega_{\mu}^{(0)}(s)-\Omega_{\mu}^{(0)}(s-\hat{\mu})\right],
$$

where $f(s, \mu)$ are the faces

$$
f(s, \mu)=c(s) \cap c(s-\hat{\mu}),
$$

which have the orientation of $\partial c(s)$, and $\hat{\mu}$ is the unit vector in the $\mu$-direction.

Following the discussion in the previous section we can write

$$
\Omega_{\mu}^{(0)}(s)-\Omega_{\mu}^{(0)}(s-\hat{\mu})=\Delta \Omega_{\mu}^{(0)}(s, s-\hat{\mu})=\partial_{\nu} \Omega_{\mu \nu}^{(1)}(s, s-\hat{\mu})
$$

except for a set of singular points $x \in f(s, \mu) \backslash \partial f(s, \mu)$. As before the topological charge receives contributions from two terms, $Q=Q^{(1)}+Q_{\Sigma}^{(1)}$, where now

$$
Q_{\Sigma}^{(1)}=\sum_{s \in A} \sum_{\mu, v} \int_{p(s, \mu, v)} d^{2} \sigma_{\mu \nu}\left[\Omega_{\mu \nu}^{(1)}(s, s-\hat{\mu})-\Omega_{\mu \nu}^{(1)}(s-\hat{v}, s-\hat{\mu}-\hat{v})\right]
$$


with

$$
p(s, \mu, v)=c(s) \cap c(s-\hat{\mu}) \cap c(s-\hat{v})
$$

being a plaquette, which was the orientation of $\partial f(s, \mu)$. The contribution $Q^{(1)}$, which is independent of the particular geometry, is precisely the same as in the previous case,

$$
Q^{(1)}=\sum_{\Lambda^{(1)}} n^{(1)}(x ; s, s-\hat{\mu}),
$$

where the sum is over all singular points

$$
\Lambda^{(1)}=\bigcup_{s, \mu}\{x \in f(s, \mu) \backslash \partial f(s, \mu)\} .
$$

Noticing that [cf. Eq. (15)]

$$
\begin{gathered}
\Omega_{\mu \nu}^{(1)}(s, s-\hat{\mu}-\hat{v})=-\Omega_{v \mu}^{(1)}(s, s-\hat{\mu}-\hat{v}), \\
\Omega_{\mu \nu}^{(1)}(s-\hat{v}, s-\hat{\mu}-\hat{v})=-\Omega_{v \mu}^{(1)}(s-\hat{v}, s-\hat{\mu}-\hat{v}),
\end{gathered}
$$

$Q_{\Sigma}^{(1)}$ can be rewritten

$$
\begin{aligned}
Q_{\Sigma}^{(1)} & =\sum_{s \in \Lambda} \sum_{\mu, v} \int_{p(s, \mu, v)} d^{2} \sigma_{\mu \nu}\left[\Omega_{\mu \nu}^{(1)}(s, s-\hat{\mu})-\Omega_{\mu \nu}^{(1)}(s, s-\hat{\mu}-\hat{v})+\Omega_{\mu \nu}^{(1)}(s-\hat{\mu}, s-\hat{\mu}-\hat{v})\right] \\
& =\sum_{s \in \Lambda} \sum_{\mu, v} \int_{p(s, \mu, v)} d^{2} \sigma_{\mu \nu} \Delta \Omega_{\mu \nu}^{(1)}(s, s-\hat{\mu}, s-\hat{\mu}-\hat{v}) \\
& =\sum_{s \in \Lambda} \sum_{\mu, v} \sum_{p(s, \mu, v)} d^{2} \sigma_{\mu \nu} \partial_{\varrho} \Omega_{\mu v \varrho}^{(2)}(s, s-\hat{\mu}, s-\hat{\mu}-\hat{v})
\end{aligned}
$$

which gives as before $Q_{\Sigma}^{(1)}=Q^{(2)}+Q_{\Sigma}^{(2)}$, where

$$
\begin{aligned}
Q_{\Sigma}^{(2)}= & \sum_{s \in \Lambda} \sum_{\mu, v, \varrho} \int_{l(s, \mu, v, \varrho)} d \sigma_{\mu v \varrho}\left[\Omega_{\mu \nu \varrho}^{(2)}(s, s-\hat{\mu}, s-\hat{\mu}-\hat{v})\right. \\
& \left.-\Omega_{\mu v \varrho}^{(2)}(s-\hat{\varrho}, s-\hat{\mu}-\hat{\varrho}, s-\hat{\mu}-\hat{v}-\hat{\varrho})\right]
\end{aligned}
$$

with

$$
l(s, \mu, v, \varrho)=c(s) \cap c(s-\hat{\mu}) \cap c(s-\hat{v}) \cap c(s-\hat{\varrho})
$$

being a link, which has the orientation of $\partial p(s, \mu, v)$. The contribution $Q^{(2)}$ has the form

$$
Q^{(2)}=\sum_{\Lambda^{(2)}} n^{(2)}(x ; s, s-\hat{\mu}, s-\hat{\mu}-\hat{v}),
$$

where

$$
\Lambda^{(2)}=\bigcup_{s, \mu, v}\{x \in p(s, \mu, v) \backslash \partial p(s, \mu, v)\} .
$$

Making use of the fact that [9]

$$
\begin{aligned}
\Omega_{\mu \nu \varrho}^{(2)}(\mathrm{s}, \mathrm{s}-\hat{\mu}, \mathrm{s}-\hat{\mu}-\hat{v}-\hat{\varrho}) & =-\Omega_{\mu \varrho \nu}^{(2)}(s, s-\hat{\mu}, s-\hat{\mu}-\hat{v}-\hat{\varrho}), \\
\Omega_{\mu \nu \varrho}^{(2)}(s, s-\hat{\mu}-\hat{v}, s-\hat{\mu}-\hat{v}-\hat{\varrho}) & =-\Omega_{v \mu \varrho}^{(2)}(s, s-\hat{\mu}-\hat{v}, s-\hat{\mu}-\hat{v}-\hat{\varrho}), \\
\Omega_{\mu \nu \varrho}^{(2)}(s-\hat{\varrho}, s-\hat{\mu}-\hat{\varrho}, s-\hat{\mu}-\hat{v}-\hat{\varrho}) & =\Omega_{\varrho \mu \nu}^{(2)}(s-\hat{\varrho}, s-\hat{\mu}-\hat{\varrho}, s-\hat{\mu}-\hat{v}-\hat{\varrho}),
\end{aligned}
$$


Eq. (68) can be written

$$
\begin{aligned}
Q_{\Sigma}^{(2)}= & \sum_{s \in \Lambda} \sum_{\mu, v, \varrho} \int_{l(s, \mu, v, \varrho)} d \sigma_{\mu \nu \varrho} \\
& \cdot\left[\Omega_{\mu \nu \varrho}^{(2)}(s, s-\hat{\mu}, s-\hat{\mu}-\hat{v})-\Omega_{\mu v \varrho}^{(2)}(s, s-\hat{\mu}, s-\hat{\mu}-\hat{v}-\hat{\varrho})\right. \\
& \left.+\Omega_{\mu \nu \varrho}^{(2)}(s, s-\hat{\mu}-\hat{v}, s-\hat{\mu}-\hat{v}-\hat{\varrho})-\Omega_{\mu \nu \varrho}^{(2)}(s-\hat{\mu}, s-\hat{\mu}-\hat{v}, s-\hat{\mu}-\hat{v}-\hat{\varrho})\right] \\
= & \sum_{s \in \Lambda} \sum_{\mu, v, \varrho} \int_{l(s, \mu, v, \varrho)} d \sigma_{\mu \nu \varrho} \Delta \Omega_{\mu v \varrho}^{(2)}(s, s-\hat{\mu}, s-\hat{\mu}-\hat{v}, s-\hat{\mu}-\hat{v}-\hat{\varrho}) \\
= & \sum_{s \in \Lambda} \sum_{\mu, v, \varrho} \int_{l(s, \mu, v, \varrho)} d \sigma_{\mu \nu \varrho} \partial_{\sigma} \Omega_{\mu \nu \varrho \sigma}^{(3)}(s, s-\hat{\mu}, s-\hat{\mu}-\hat{v}, s-\hat{\mu}-\hat{v}-\hat{\varrho}) .
\end{aligned}
$$

This gives, as before, $Q_{\Sigma}^{(2)}=Q^{(3)}+Q_{\Sigma}^{(3)}$, where

$$
Q^{(3)}=\sum_{\Lambda^{(3)}} n^{(3)}(x ; s, s-\hat{\mu}, s-\hat{\mu}-\hat{v}, s-\hat{\mu}-\hat{v}-\hat{\varrho})
$$

with

$$
\Lambda^{(3)}=\bigcup_{s, \mu, v, \varrho}\{x \in l(s, \mu, v, \varrho) \backslash \partial l(s, \mu, v, \varrho)\} .
$$

For $Q_{\Sigma}^{(3)}$ we obtain, together with Eq. (40),

$$
\begin{aligned}
Q_{\Sigma}^{(3)}= & \sum_{s \in \Lambda} \sum_{\mu, v, \varrho, \sigma} \varepsilon_{\mu \nu \varrho \sigma} \frac{1}{2 \pi^{2}}[V(s, s-\hat{\mu}, s-\hat{\mu}-\hat{v}, s-\hat{\mu}-\hat{v}-\hat{\varrho}) \\
& -V(s-\hat{\sigma}, s-\hat{\mu}-\hat{\sigma}, s-\hat{\mu}-\hat{v}-\hat{\sigma}, s-\hat{\mu}-\hat{v}-\hat{\varrho}-\hat{\sigma})],
\end{aligned}
$$

where $V(s, s-\hat{\mu}, s-\hat{\mu}-\hat{v}, s-\hat{\mu}-\hat{v}-\hat{\varrho}), \ldots$ are the volumes of the spherical tetrahedra defined by the transition functions $v_{s, s-\mu}, \ldots$.

Again, making use of the symmetry/antisymmetry properties of $\varepsilon_{\mu \nu \varrho \sigma}$, Eq. (76) can be written

$$
\begin{aligned}
Q_{\Sigma}^{(3)}= & \sum_{s \in \Lambda} \sum_{\mu, v, \varrho, \sigma} \varepsilon_{\mu \nu \varrho \sigma} \frac{1}{2 \pi^{2}}[V(s, s-\hat{\mu}, s-\hat{\mu}-\hat{v}, s-\hat{\mu}-\hat{v}-\hat{\varrho}) \\
& -V(s, s-\hat{\mu}, s-\hat{\mu}-\hat{v}, s-\hat{\mu}-\hat{v}-\hat{\varrho}-\hat{\sigma}) \\
& +V(s, s-\hat{\mu}, s-\hat{\mu}-\hat{v}-\hat{\varrho}, s-\hat{\mu}-\hat{v}-\hat{\varrho}-\hat{\sigma}) \\
& -V(s, s-\hat{\mu}-\hat{v}, s-\hat{\mu}-\hat{v}-\hat{\varrho}, s-\hat{\mu}-\hat{v}-\hat{\varrho}-\hat{\sigma}) \\
& +V(s-\hat{\mu}, s-\hat{\mu}-\hat{v}, s-\hat{\mu}-\hat{v}-\hat{\varrho}, s-\hat{\mu}-\hat{v}-\hat{\varrho}-\hat{\sigma})],
\end{aligned}
$$

so that finally we obtain

$$
Q_{\Sigma}^{(3)}=\sum_{s \in \Lambda} \sum_{\mu, v, \varrho, \sigma} n(s, s-\hat{\mu}, s-\hat{\mu}-\hat{v}, s-\hat{\mu}-\hat{v}-\hat{\varrho}, s-\hat{\mu}-\hat{v}-\hat{\varrho}-\hat{\sigma}),
$$

where $n$ is the winding number associated with the 5 spherical tetrahedra covering $S^{3}$ as introduced before with values $0, \pm 1$.

In total, the topological charge then reads

$$
\begin{aligned}
Q= & \sum_{s \in \Lambda} \sum_{\mu, v, \varrho, \sigma} n(s, s-\hat{\mu}, s-\hat{\mu}-\hat{v}, s-\hat{\mu}-\hat{v}-\hat{\varrho}, s-\hat{\mu}-\hat{v}-\hat{\varrho}-\hat{\sigma}) \\
& +\sum_{\Lambda^{(1)}} n^{(1)}(x ; s, s-\hat{\mu})+\sum_{\Lambda^{(2)}} n^{(2)}(x ; s, s-\hat{\mu}, s-\hat{\mu}-\hat{v}) \\
& +\sum_{\Lambda^{(3)}} n^{(3)}(x ; s, s-\hat{\mu}, s-\hat{\mu}-\hat{v}, s-\hat{\mu}-\hat{v}-\hat{\varrho}) .
\end{aligned}
$$


Again, it can be arranged that

$$
Q=\sum_{s \in \Lambda} \sum_{\mu, v, \varrho, \sigma} n(s, s-\hat{\mu}, s-\hat{\mu}-\hat{v}, s-\hat{\mu}-\hat{v}-\hat{\varrho}, s-\hat{\mu}-\hat{v}-\hat{\varrho}-\hat{\sigma}) .
$$

The virtue of this construction of the topological charge $Q$ is that it can easily be adopted to any geometry of cells.

\section{Lattice Gauge Fields}

In Sects. II and III we have assumed that the gauge fields are known in the continuum (of $\mathbb{M}$ ). This is no longer true if the theory is regularized by formulating it on a discrete lattice of points in space-time - usually a hypercubic lattice, which we shall identify with $\Lambda$ [Eq. (54)]. In this case the transition functions $v_{s, s-\hat{\mu}}$ are a priori only given at the corners of the faces $f(s, \mu)$, i.e. the lattice points.

For the computation of $Q_{\Sigma}^{(3)}$ this is all that is required. For the computation of $Q^{(1)}, Q^{(2)}$, and $Q^{(3)}$ we need - if no further provisions are made - to know whether $\Delta \Omega_{\mu}^{(0)}(s, s-\hat{\mu}), \Delta \Omega_{\mu \nu}^{(1)}(s, s-\hat{\mu}, s-\hat{\mu}-\hat{v})$, and $\Delta \Omega_{\mu \nu \varrho}^{(2)}(s, s-\hat{\mu}, s-\hat{\mu}-\hat{v}, s-\hat{\mu}-\hat{v}-\hat{\varrho})$ are singular in $f(s, \mu) \backslash \partial f(s, \mu), p(s, \mu, v) \backslash \partial p(s, \mu, v)$, and $l(s, \mu, v, \varrho) \backslash \partial l(s, \mu, v, \varrho)$, respectively. The exact positions of the singularities $\left(x \in \Lambda^{(1)}, \Lambda^{(2)}\right.$, and $\Lambda^{(3)}$, respectively) are, however, not necessary to know. This requires (only) an interpolation of $v_{s, s-\hat{\mu}}$ throughout the plaquettes $p(s, \mu, v)$ and, possibly, to a single (but arbitrary) point in the interior of $f(s, \mu)$. A potential interpolation (which satisfies the cocycle condition and maintains gauge invariance) has been given in the literature [8].

In the "continuum region," where the lattice spacing (which we have set to 1) becomes small in physical units and the gauge fields contributing to the functional integral have a small (lattice) action density,

$$
\operatorname{Tr}[1-U(\partial p)]<\varepsilon
$$

$[U(\partial p):$ parallel transporter around the plaquette $p]$, the computation of $Q=Q_{\Sigma}^{(3)}$ $+Q^{(1)}+Q^{(2)}+Q^{(3)}$ simplifies greatly. Using Lüscher's interpolation [8] of the transition functions, one derives the following (sufficient) conditions under which $\Delta \Omega_{\mu}^{(0)}(s, s-\hat{\mu}), \Delta \Omega_{\mu \nu}^{(1)}(s, s-\hat{\mu}, s-\hat{\mu}-\hat{v})$, and $\Delta \Omega_{\mu \nu \varrho}^{(2)}(s, s-\hat{\mu}, s-\hat{\mu}-\hat{v}, s-\hat{\mu}-\hat{v}-\hat{\varrho})$ have no gauge singularities (assuming $\left.14 \arccos \left(1-\frac{1}{2} \varepsilon\right) \leqq \pi\right)$ :

$$
\begin{gathered}
d\left(v_{s, s-\hat{\mu}}(s), 1\right)+11 \arccos \left(1-\frac{1}{2} \varepsilon\right) \leqq \pi, \\
d\left(v_{s, s-\mu}(s), 1\right)+d\left(v_{s-\hat{\mu}, s-\hat{\mu}-\hat{v}}(s), 1\right)+d\left(v_{s, s-\hat{\mu}}(s) v_{s-\hat{\mu}, s-\hat{\mu}-\hat{v}}(s), 1\right) \\
+6 \arccos \left(1-\frac{1}{2} \varepsilon\right) \leqq 2 \pi, \\
d\left(v_{s, s-\hat{\mu}}(s), 1\right)+\max _{0 \leqq \lambda \leqq 1}\left\{d\left(v_{s-\hat{\mu}, s-\hat{\mu}-\hat{v}}(s)\left[v_{s-\hat{\mu}-\hat{v}, s-\hat{\mu}-\hat{v}-\hat{\varrho}}(s)\right]^{\lambda}, 1\right)\right. \\
\left.+d\left(v_{s, s-\hat{\mu}}(s) v_{s-\hat{\mu}, s-\hat{\mu}-\hat{v}}(s)\left[v_{s-\hat{\mu}-\hat{v}, s-\hat{\mu}-\hat{v}-\hat{\varrho}}(s)\right]^{\lambda}, 1\right)\right\} \\
+8 \arccos \left(1-\frac{1}{2} \varepsilon\right) \leqq 2 \pi
\end{gathered}
$$

for $\mu, v, \varrho \in\{2,3,4\}$ (for $\mu, v$ or $\varrho=1$ the fields that give rise to gauge singularities are of measure zero in the functional integral), where $d(u, v)$ is the metric of $\mathrm{SU}(2)=S^{3}$, i.e.

$$
d(u, 1)=\arccos \left(\frac{1}{2} \operatorname{Tr} u\right)
$$


This means that for small $\varepsilon$ it is possible to gauge transform the transition functions - if necessary - such that the bounds (82) are satisfied. The computation of $Q$ then reduces to the computation of $Q_{\Sigma}^{(3)}$, and the charge can be viewed as the sum over local SU(2) winding numbers.

For gauge field configurations with larger action density it is also not necessary to compute all of the $Q$ 's. One finds that it is always possible to (explicitly) gauge transform the transition functions such that $Q_{\Sigma}^{(3)}=Q^{(2)}=Q^{(1)}=0$. This then reduces the problem to determine whether $v_{s, s-\hat{\mu}}=-1$ in $f(s, \mu) \backslash \partial f(s, \mu)$, which can be done on purely geometrical grounds.

It is not the purpose of the present paper to go into further details of the numerical evaluation of $Q$ on the lattice. This will be the subject of a forthcoming publication, where we will also present results of a Monte Carlo calculation of $\left\langle Q^{2}\right\rangle$ on large lattices.

\section{SU(3)}

It is not trivial to extend the results of Sects. II and III to the physically more interesting case of gauge group SU(3). Therefore one is led to ask the question: can we reduce the problem of computing the topological charge for SU(3) gauge fields to the case of $\mathrm{SU}(2)[10]$ ?

In the continuum this is possible by means of the so-called reduction of the structure group [11]. This means the following. A fibre bundle, which has structure group $G$ and is therefore in general given by $G$-valued transition functions, may under certain circumstances be described by transition functions with values in a subgroup of $G$. By means of the theorems proved in [11] it is easily shown that the structure group of any $\mathrm{SU}(3)$ bundle over $T^{4}$ can be reduced to $\mathrm{SU}(2)$.

The explicit construction makes use of the fact that $\mathrm{SU}(3) / \mathrm{SU}(2)$ is equivalent to $S^{5}$. Actually, $\mathrm{SU}(3)$ is a $\mathrm{SU}(2)$ principal bundle over $S^{5}$ (cf. [12] for the analogous considerations in case of the unitary groups). The projection

$$
\mathrm{SU}(3) \rightarrow \mathrm{SU}(3) / \mathrm{SU}(2) \cong S^{5}
$$

simply maps a SU(3) matrix onto its first column. Here and in the following we describe points of $S^{5}$ by a triplet of complex numbers,

$$
(a, b, c) \in \mathbb{C}^{3}, \quad|a|^{2}+|b|^{2}+|c|^{2}=1 .
$$

As $S^{5}$ minus one point is a contractible space, $\mathrm{SU}(3)$ minus the fibre over the removed point is a trivial $\mathrm{SU}(2)$ bundle. For example, any $u \in \mathrm{SU}(3)$ with $u_{11} \neq-1$ may be decomposed as

where $|\alpha|^{2}+|\beta|^{2}=1$ and

$$
u=\omega\left(u_{11}, u_{21}, u_{31}\right)\left(\begin{array}{ccc}
1 & 0 & 0 \\
0 & \alpha & -\beta^{*} \\
0 & \beta & \alpha^{*}
\end{array}\right)
$$

$$
\omega(a, b, c)=\left(\begin{array}{ccc}
a & -b^{*} & -c^{*} \frac{1+a}{1+a^{*}} \\
b & \frac{1}{1+a}\left(1+a^{*}-|b|^{2}\right) & -\frac{b c^{*}}{1+a^{*}} \\
c & -\frac{c b^{*}}{1+a} & \frac{1}{1+a^{*}}\left(1+a^{*}-|c|^{2}\right)
\end{array}\right)
$$


for $|a|^{2}+|b|^{2}+|c|^{2}=1, a \neq-1$. The first factor in (86) represents a point of the base space $S^{5}$ in SU(3) while the second one describes the fibre $\operatorname{SU}(2)$.

Now the reduction procedure as given in [11] goes as follows. Let $\mathscr{P}$ be an $\mathrm{SU}(3)$ bundle over $T^{4}$ specified by transition functions (we shall restrict ourselves here to hypercubic geometry)

$$
\bar{v}_{s, s-\hat{\mu}}(y) \in \mathrm{SU}(3), \quad y \in f(s, \mu)
$$

analogous to the case of SU(2). We first construct a section of the bundle $\mathscr{P} / \mathrm{SU}(2)$ which has $T^{4}$ as base space and $S^{5}$ as fibre. Such a section is described by smooth functions

$$
z_{s}: c(s) \rightarrow S^{5}
$$

satisfying

$$
z_{s}(y)=\bar{v}_{s, s-\hat{\mu}}(y) z_{s-\hat{\mu}}(y)
$$

for $y \in f(s, \mu)$. The fact that $\mathscr{P} / \mathrm{SU}(2)$ admits a section follows from

$$
\pi_{1}\left(S^{5}\right)=\pi_{2}\left(S^{5}\right)=\pi_{3}\left(S^{5}\right)=0 .
$$

If $z_{s}$ is not surjective - this condition is generically fulfilled - we can find a smooth map

$$
\hat{z}_{s}: c(s) \rightarrow \mathrm{SU}(3)
$$

such that the first column of $\hat{z}_{s}(y)$ coincides with $z_{s}(y)$ for all $y \in c(s)$ :

$$
\hat{z}_{s}(y)\left(\begin{array}{l}
1 \\
0 \\
0
\end{array}\right)=z_{s}(y) \text {. }
$$

For example, if $z_{s}(y) \neq(-1,0,0)$ for all $y \in c(s)$ we can define

$$
\hat{z}_{s}(y)=\omega\left(z_{s}(y)\right) \text {. }
$$

For $y \in f(s, \mu)$, we have

$$
\hat{z}_{s}(y)\left(\begin{array}{l}
1 \\
0 \\
0
\end{array}\right)=z_{s}(y)=\bar{v}_{s, s-\hat{\mu}}(y) z_{s-\hat{\mu}}(y)=\bar{v}_{s, s-\hat{\mu}}(y) \hat{z}_{s-\hat{\mu}}(y)\left(\begin{array}{l}
1 \\
0 \\
0
\end{array}\right) .
$$

Consequently, if we use the functions $\hat{z}_{s}$ to gauge transform the $\bar{v}_{s, s-\hat{\mu}}$, we arrive at transition functions with values in $\mathrm{SU}(2)$ :

$$
\hat{z}_{s}(y)^{-1} \bar{v}_{s, s-\hat{\mu}}(y) \hat{z}_{s-\hat{\mu}}(y)=\left(\begin{array}{ccc}
1 & 0 & 0 \\
0 & & \\
0 & & v_{s, s-\hat{\mu}}
\end{array}\right), \quad v_{s, s-\hat{\mu}}(y) \in \operatorname{SU}(2) .
$$

As the topological charge is invariant under gauge transformations like (96), it can be computed from the $\mathrm{SU}(2)$-valued transition functions $v_{s, s-\hat{\mu}}(y)$.

To carry out the reduction explicitly we need to know $z_{s}(y)$. In the continuum, where the SU(3)-valued transition functions $\bar{v}_{s, s-\hat{\mu}}$ are explicitly given in the form

$$
\bar{v}_{s, s-\hat{\mu}}(y)=\bar{g}_{s}(y)^{-1} \bar{g}_{s-\mu}(y)
$$


[cf. Eq. (11)] for all $y \in f(s, \mu)$, we may take

$$
z_{s}(y)=\bar{g}_{s}(y)^{-1}\left(\begin{array}{l}
1 \\
0 \\
0
\end{array}\right)
$$

which, as is easily seen, satisfies Eq. (90) (and, as a result of (91), can be extended to $c(s)$ ). On the lattice we follow [8] and construct transition functions at the corners of $f(s, \mu)$ with help of the standard parallel transporters $\bar{w}^{s}$ :

$$
\bar{v}_{s, s-\mu}(y)=\bar{w}^{s}(y) \bar{w}^{s-\hat{\mu}}(y)^{-1} .
$$

To extend the $\bar{v}_{s, s-\hat{\mu}}$ 's to the whole of $f(s, \mu)$ we may take Lüscher's interpolation [8] (which is trivial to extend to the case of gauge group SU(3)). It has been shown in [13] that this can be written for all $y \in f(s, \mu)$ in the form (99). The explicit expressions for the parallel transporters $\bar{w}^{s}(y), y \in \partial c(s)$ are also given in this reference. We then may define

$$
z_{s}(y)=\bar{w}^{s}(y)\left(\begin{array}{l}
1 \\
0 \\
0
\end{array}\right),
$$

which satisfies Eq. (90) and provides us with SU(2)-valued transition functions $v_{s, s-\hat{\mu}}(y)$ for all $y \in f(s, \mu)$.

\section{Conclusions}

We have integrated the Chern-Pontryagin density completely and obtained a closed expression for the topological charge $Q$ - which requires one to know the gauge singularities of $\Delta \Omega_{\mu}^{(0)}, \Delta \Omega_{\mu \nu}^{(1)}$, and $\Delta \Omega_{\mu \nu \varrho}^{(2)}$ though. This result applies as well for gauge group SU(3), whose transition functions can be gauge transformed into transition functions with values in $\mathrm{SU}(2)$. The practical use of the expression is that it allows us to calculate $Q$, in the continuum and on the lattice, without resort to numerical integration.

We have seen that Eq. (79) leaves considerable room for the practical evaluation of $Q$ on the lattice - including the choice of an interpolation of the transition functions. The by far fastest procedure is the first method described in Sect. IV, in which it is sufficient to compute $Q_{\Sigma}^{(3)}$. This will be applicable in the "continuum region" only. But notice that alone in the "continuum region" a lattice gauge field configuration can be assigned a unique topological charge [8].

While we were preparing this paper we received a preprint by Phillips and Stone [14], who also were able to compute the topological charge of SU(2) gauge fields by analytical means. The amount of algebra to do in their algorithm is similar to that of the second method sketched in Sect. IV, where one has to compute $Q^{(1)}$ only. It will be interesting to compare their charge with, e.g., Lüscher's interpolation of the transition functions on individual gauge field configurations at various values of the coupling constant to test for uniqueness of the results. 


\section{References}

1. Belavin, A.A., Polyakov, A.M., Schwartz, A.S., Tyupkin, Yu.S.: Phys. Lett. 59B, 85 (1975)

2. Fox, I.A., Gilchrist, J.P., Laursen, M.L., Schierholz, G.: Phys. Rev. Lett. 54, 749 (1985)

3. Arian, Y., Woit, P.: Nucl. Phys. B 268, 521 (1986)

4. 't Hooft, G.: Phys. Rev. Lett. 37, 8 (1976); Phys. Rev. D 14, 3432 (1976)

5. Illgenfritz, E.-M., Laursen, M.L., Müller-Preußker, M., Schierholz, G., Schiller, H.: Nucl. Phys. B 268, 693 (1986)

6. Callan, C., Dashen, R., Gross, D.: Phys. Rev. D 19, 1826 (1979) Shuryak, E.V.: Phys. Rep. 115, 151 (1984)

7. Callan, C., Dashen, R., Gross, D.: Phys. Rev. D 17, 2717 (1978)

8. Lüscher, M.: Commun. Math. Phys. 85, 39 (1982)

9. Laursen, M.L., Schierholz, G., Wiese, U.-J.: Commun. Math. Phys. 103, 693 (1986)

10. Parisi, G., Rapuano, F.: Phys. Lett. 152B, 218 (1985)

11. Choquet-Bruhat, Y., DeWitt-Morette, C., Dillard-Bleick, M.: Analysis, manifolds, and physics. Amsterdam: North-Holland 1982, pp. 381-385

12. Steenrod, N.: The topology of fibre bundles. Princeton NJ: Princeton University Press 1951

13. Wiese, U.-J.: Thesis (to be published)

14. Phillips, A., Stone, D.: Commun. Math. Phys. 103, 599 (1986)

Communicated by G. Mack

Received February 6, 1986; in revised form June 4, 1986 
